\title{
COVID-19 outbreak impact on emergency trauma visits and trauma surgery in a level 3 trauma center
}

\author{
Buğra İlhan ${ }^{1}\left[\right.$ · Göksu Bozdereli Berikol ${ }^{1} \cdot$ Hakan Aydın ${ }^{1} \cdot$ Müge Arslan Erduhan ${ }^{2} \cdot$ Halil Doğan $^{1}$
}

Received: 3 September 2021 / Accepted: 26 September 2021 / Published online: 7 October 2021

(c) The Author(s), under exclusive licence to Royal Academy of Medicine in Ireland 2021

\begin{abstract}
Background This study aims to compare emergency trauma visits' severity, emergency surgical needs, and characteristics between the pandemic and pre-pandemic periods.

Methods This retrospective observational study was conducted in a tertiary training and research hospital between 1 and 30 April 2020 (pandemic group) and compared with the previous year's same dates (pre-pandemic group). Trauma patients aged 18 and over were included in the study. Emergency Severity Index (ESI) levels, trauma surgery needs, and injury characteristics were compared.

Results A total of 2097 patients (592 pandemic and 1505 pre-pandemic) were included. There was an approximately $60 \%$ reduction in total and daily visits. ESI levels $1(0.2 \%$ vs. $1.4 \%)$ and $2(0.8 \%$ vs. $1.9 \%)$ patients increased during pandemic period. Trauma surgery needs (1.6\% vs. $2.2 \%)$, intensive care unit (ICU) admission (0.4\% vs. $0.2 \%)$, and ward admission (6.3\% vs. $7.9 \%)$ did not change during pandemic period.

Conclusion Despite the decrease in the visit frequency of adult trauma patients during the pandemic period, the needs for trauma surgery, ICU, and ward admission did not change. Trauma teams should continue their duties during the pandemic period.
\end{abstract}

Keywords COVID-19 $\cdot$ Emergency surgery $\cdot$ Pandemic $\cdot$ Trauma $\cdot$ Wounds and injuries

\section{Introduction}

In December 2019, unusual pneumonia cases began to appear in Wuhan, China, and a new coronavirus species was detected as the causative agent. Later, this virus was named COVID-19 and was declared a pandemic by the World Health Organization (WHO) due to its rapid spread [1]. Over 216 million confirmed cases and over 4.4 million deaths had been reported so far [2]. Treatment and vaccination studies are still ongoing for this rapidly spreading disease which has high morbidity and mortality.

As the number of cases increased rapidly, all countries took some measures to protect their citizens and prevent

Buğra İlhan

bugra_ilhan@yahoo.com

1 Department of Emergency, University of Health Sciences, Bakırköy Dr. Sadi Konuk Training and Research Hospital, Istanbul, Turkey

2 Department of Emergency, University of Health Sciences, Haseki Training and Research Hospital, Istanbul, Turkey the spread of the disease. However, both the disease itself and its indirect effects had negative consequences on health systems and patients. Emergency department (ED) presentations of all patients, especially critically ill patients, have decreased during the pandemic period [3]. Also, there have been delays in the admission and treatment of surgical emergencies $[4,5]$. During the pandemic period, decreases have been reported in acute coronary syndrome and acute stroke patients [6-8]. Similarly, decreases were reported in orthopedic and pediatric trauma cases during the pandemic period [9-12].

Emergency departments have been the first presentation areas of patients during the pandemic period as well as in the pre-pandemic period. When the first COVID-19 case was seen in Turkey in the second week of March, COVID19 patients started to present to EDs in addition to nonCOVID patients. At the same time, since it is a tertiary trauma center, trauma patients continued to present to our ED. Studies evaluating trauma cases during the pandemic period were mostly conducted for a specific region or a 
specific specialty. Studies evaluating all traumas and emergency surgeries together are limited.

This study aims to compare adult trauma visits' severity, emergency surgical needs, and characteristics between the pandemic and pre-pandemic periods.

\section{Methods}

\section{Study design}

This retrospective observational study was conducted in a tertiary training and research hospital after the approval of the local ethics committee (Approval ID: 2021/56). The ED where the study was conducted is also a tertiary trauma center with approximately 350,000 applications annually in Turkey's most populous city, Istanbul. It is also a primary percutaneous coronary intervention and thrombolytic center. After the first COVID-19 case was seen on March 11, 2020 in our country, patients with suspected COVID-19 started to be accepted to the study center along with non-COVID patients.

\section{Patient selection and groups}

Adult trauma patients aged 18 years and over and presented to the ED between 1 and 30 April 2020 (pandemic group) were included in the study. Patients who transferred from another center and whose medical records could not be reached were excluded from the study. To reflect the prepandemic period and make the comparison reliable, patients on the same period of the previous year (1-30 April 2019) were included as the control group (pre-pandemic group). The same inclusion and exclusion criteria were used for the control group as well. The flow diagram of the study is shown in Fig. 1.

The data of the patients included in the study were obtained from electronic medical records and ED files. Patients' demographic information, way of arrival, injury type and region, trauma and emergency surgery needs, accident location, presence and location of fractures, consulted specialties, ED length of stay (LOS), and dispositions were recorded.

Emergency surgery need was defined as urgent surgeries within $1 \mathrm{~h}$ of arrival to the ED. Trauma surgery need was defined as surgeries that were done within $24 \mathrm{~h}$ of arrival. Elective surgery needs of the patients were not recorded since not the aim of the study.

The first evaluation of the patients was made by a registered nurse who received triage training given by the Ministry of Health in the triage field. The severity levels of the patients were determined by the Emergency Severity Index

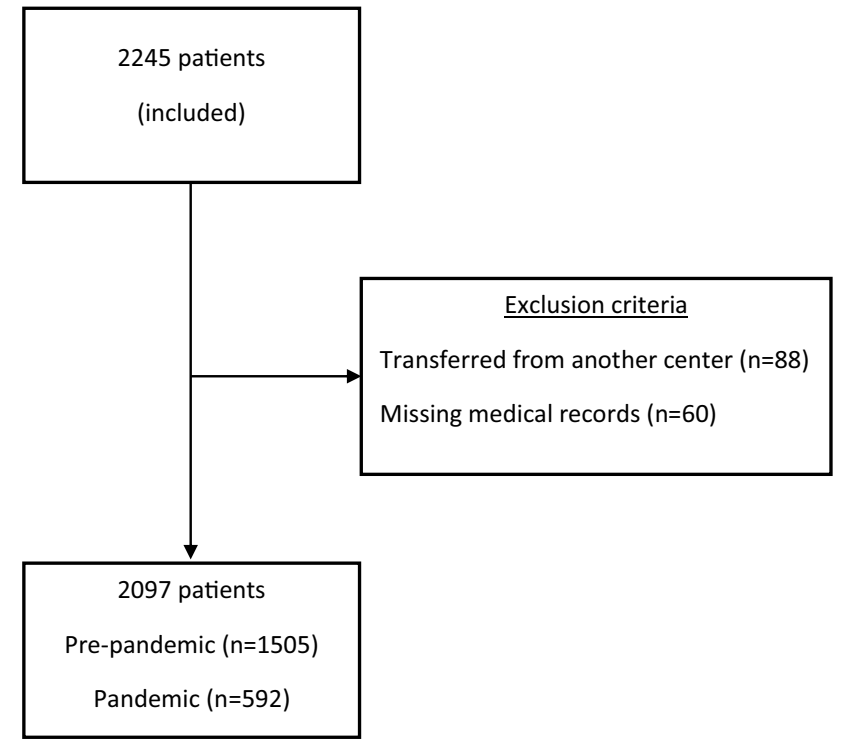

Fig. 1 Flow diagram of the study

(ESI), which achieved successful results in the triage of adult patients [13].

\section{Outcomes}

The primary outcomes of the study were the change in emergency visits and severity levels of adult trauma patients during the COVID-19 outbreak. Secondary outcomes are the changes in patients' emergency surgical needs, ward and intensive care unit (ICU) admissions, injury types, regions, and ED LOS.

\section{Statistical analysis}

Quantitative variables were presented as mean \pm standard deviation or median (IQR: interquartile range). The relationship between quantitative variables was evaluated by independent $t$-test or Mann-Whitney $U$ test. Categorical variables were presented with numbers and percentages. The relationship between categorical variables was evaluated by the chi-square test. The distribution of the groups was determined by the Shapiro-Wilks or Kolmogorov Smirnov test. Spearman's or Pearson's test was used in correlation analysis according to the distribution of the data. In univariate analysis, variables with a $p$-value less than 0.05 , sample size greater than 20 , and not correlating with each other were included in the multivariate analyzes. Odds ratios are presented with a $95 \%$ confidence interval. SPSS $®$ for Windows version 23.0 program (IBM, Chicago, IL, USA) was used for statistical analysis. Statistical significance level was accepted as $p<0.05$. 


\section{Results}

A total of 2097 patients (592 in the pandemic group and 1505 in the pre-pandemic group) were included in the analysis, and 725 (34.6\%) of them were women. The median age of the patients was 35 (IQR: 25-49). Age and gender of the patients were similar between the pandemic and pre-pandemic periods $(p>0.05)$.

Approximately $60 \%$ reduction was found in trauma patients' total and daily visits during the pandemic period. The frequency of critically ill trauma patient visits at ESI levels 1 and 2 increased significantly during the pandemic. There were no changes in the trauma and emergency surgery needs, ward, and ICU admissions. Patients preferred to arrive at the ED by ambulance at a higher rate during the pandemic period $(p<0.001)$. The ED LOS did not change in the pandemic period. The demographic data, ESI levels, and disposition of the patients are shown in Table 1.

When the injury characteristics were evaluated, the frequency of blunt trauma decreased significantly during the pandemic period. Falls from height and motorcycle accidents increased significantly. While the frequency of multiple trauma decreased, the frequency of upper and lower limb injuries increased $(p<0.05)$. When accident locations were evaluated, there was no significant change in in-home injuries, but the frequency of street injuries decreased $(p=0.015)$. The injury characteristics of the patients during pre-pandemic and pandemic periods are shown in Table 2.

The majority of the ESI level 1 patients were pedestrian accident $(37.5 \%)$, fall from height (25\%), and penetrating objects-related injury (25\%) during the pandemic period. The majority of the ESI level 2 patients were penetrating objects-related injury (63.6\%) and fall from ground level $(18.2 \%)$ during the pandemic period.

There was an approximately $60 \%$ reduction in the total fracture frequency during the pandemic period. While nasal fracture $(n=70,20 \%)$ was detected most frequently in the pre-pandemic period, rib fracture $(n=15,11 \%)$ was observed most frequently during the pandemic period. The fractures seen in both periods are shown in Fig. 2.

During the pandemic period, total trauma consultations requested from the ED were lower than the pre-pandemic period $(45.4 \%$ vs. $53.7 \%$ respectively) $(p=0.001)$. While orthopedics, otolaryngology, and plastic surgery consultation rates decreased significantly, the consultation rates of anesthesia and other trauma clinics (general surgery, neurosurgery, cardiovascular surgery, ophthalmology, thoracic surgery, gynecology, and urology) did not change.

Factors affecting trauma visits during the pandemic period were arrival by ambulance (OR: 1.66 (95\% CI
Table 1 Pre-pandemic and pandemic characteristics of the patients

\begin{tabular}{|c|c|c|c|}
\hline & Pre-pandemic & Pandemic & $p$ value \\
\hline Total ED visits, $n$ & 1505 & 592 & \\
\hline Daily visits, mean $\pm \mathrm{SD}$ & $50.17 \pm 17.36$ & $19.73 \pm 9.24$ & $<0.001 *$ \\
\hline \multicolumn{4}{|l|}{ Demographics } \\
\hline Female, $n(\%)$ & $539(35.8)$ & $186(31.4)$ & $0.057 * *$ \\
\hline Age, median (IQR) & $35(25-50)$ & $35(26-47)$ & $0.858 * * *$ \\
\hline \multicolumn{4}{|l|}{ Arrival, $n(\%)$} \\
\hline Ambulatory & $1183(78.6)$ & $416(70.3)$ & $<0.001 * *$ \\
\hline EMS, ambulance & $322(21.4)$ & $176(29.7)$ & \\
\hline \multicolumn{4}{|c|}{ Emergency Severity Index, $n(\%)$} \\
\hline Level 1 & $3(0.2)$ & $8(1.4)$ & $0.001 * *$ \\
\hline Level 2 & $12(0.8)$ & $11(1.9)$ & $0.036 * *$ \\
\hline Level 3 & $722(48)$ & $206(34.8)$ & $<0.001 * *$ \\
\hline Level 4 & $579(38.5)$ & $184(31.1)$ & $0.002 * *$ \\
\hline Level 5 & 189 (12.6) & $183(30.9)$ & $<0.001 * *$ \\
\hline \multicolumn{4}{|l|}{ Disposition, $n(\%)$} \\
\hline Trauma surgery & $24(1.6)$ & $13(2.2)$ & $0.347 * *$ \\
\hline Emergency surgery & $8(0.5)$ & $5(0.8)$ & $0.411 * *$ \\
\hline ICU admission & $6(0.4)$ & $1(0.2)$ & $0.412 * *$ \\
\hline Ward admission & $95(6.3)$ & $47(7.9)$ & $0.182 * *$ \\
\hline Discharge & $1297(86.2)$ & 459 (77.5) & $<0.001 * *$ \\
\hline Against medical advice & $73(4.9)$ & $72(12.2)$ & $<0.001 * *$ \\
\hline $\begin{array}{l}\text { Referral to another } \\
\text { hospital }\end{array}$ & $23(1.5)$ & $7(1.2)$ & $0.548 * *$ \\
\hline Exitus & $3(0.2)$ & $1(0.2)$ & $0.886^{* *}$ \\
\hline $\begin{array}{l}\text { ED LOS, min, median } \\
\text { (IQR) }\end{array}$ & $48(23-118)$ & $53(20-156)$ & $0.381 * * *$ \\
\hline
\end{tabular}

$E D$ emergency department, $S D$ standard deviation, $I Q R$ interquartile range, $E M S$ emergency medical service, ICU intensive care unit, $L O S$ length of stay

*Independent $t$ test; **Chi-square test; ***Mann-Whitney $U$ test; $p<0.05$ considered significant

1.27-2.18), $p<0.001)$, fall from height (OR: 3.0 (95\% CI 1.68-5.36), $p<0.001$ ), and motorcycle accident (OR: 3.43 (95\% CI 2.07-5.69), $p<0.001)$. Factors affecting adult trauma visits during the pandemic period are shown in Table 3.

\section{Discussion}

There was a $60 \%$ reduction in the total and daily visits of adult trauma patients during the pandemic period. The visits of critically ill trauma patients at ESI levels 1 and 2 increased significantly. Trauma surgery and emergency surgery needs, ICU, and ward admissions did not change.

In a study conducted by Pintado et al. at a level 1 trauma center, an approximately $80 \%$ reduction in orthopedic trauma admissions was detected [14]. In the study conducted by Göksoy et al., there was an approximately $25 \%$ decrease 
Table 2 Pre-pandemic and pandemic injury characteristics of the patients

\begin{tabular}{|c|c|c|c|}
\hline & Pre-pandemic & Pandemic & $p$ value* \\
\hline \multicolumn{4}{|l|}{ Injuries, $n(\%)$} \\
\hline Blunt trauma & $1044(69.4)$ & $323(54.6)$ & $<0.001$ \\
\hline Penetrating trauma & $425(28.2)$ & $261(44.1)$ & $<0.001$ \\
\hline Burn & $36(2.4)$ & $8(1.4)$ & 0.134 \\
\hline Occupational accident & $137(9.1)$ & $62(10.5)$ & 0.335 \\
\hline Fractures & 284 (18.9) & $103(17.4)$ & 0.434 \\
\hline Open fractures & $3(0.2)$ & $4(0.7)$ & 0.143 \\
\hline Dislocations & $8(0.5)$ & $9(1.5)$ & 0.023 \\
\hline \multicolumn{4}{|l|}{ Mechanism of injury, $n(\%)$} \\
\hline Fall from ground level & $449(29.8)$ & $160(27)$ & 0.202 \\
\hline Fall from height & $29(1.9)$ & $30(5.1)$ & $<0.001$ \\
\hline Motorcycle accident & $43(2.9)$ & $40(6.8)$ & $<0.001$ \\
\hline Motor vehicle accident & $74(4.9)$ & $19(3.2)$ & 0.087 \\
\hline Pedestrian accident & $79(5.2)$ & $12(2)$ & 0.001 \\
\hline Gunshot wounds & $16(1.1)$ & $11(1.9)$ & 0.146 \\
\hline $\begin{array}{l}\text { Penetrating object-related } \\
\text { injury }\end{array}$ & 270 (17.9) & $93(15.7)$ & 0.224 \\
\hline Sports related injury & $9(0.6)$ & $2(0.3)$ & 0.458 \\
\hline Direct blow & $87(5.8)$ & $31(5.2)$ & 0.626 \\
\hline Electrocution injury & $4(0.3)$ & $1(0.2)$ & 0.682 \\
\hline Animal bites & $17(1.1)$ & $91(15.4)$ & $<0.001$ \\
\hline Burns & $36(2.4)$ & $8(1.4)$ & 0.134 \\
\hline Bicycle accident & $7(0.5)$ & $1(0.2)$ & 0.322 \\
\hline Hit(ten by) something & $389(25.8)$ & $94(15.9)$ & $<0.001$ \\
\hline \multicolumn{4}{|l|}{ Region of injury, $n(\%)$} \\
\hline Multiple trauma & 325 (21.6) & $62(10.5)$ & $<0.001$ \\
\hline Head & $178(11.8)$ & $80(13.5)$ & 0.290 \\
\hline Maxillofacial & $275(18.3)$ & $99(16.7)$ & 0.404 \\
\hline Cervical & $8(0.5)$ & $5(0.8)$ & 0.411 \\
\hline Thorax & $112(7.4)$ & $38(6.4)$ & 0.413 \\
\hline Abdomen & $16(1.1)$ & $7(1.2)$ & 0.813 \\
\hline Upper limb & $341(22.7)$ & $162(27.4)$ & 0.023 \\
\hline Lower limb & $177(11.8)$ & $112(18.9)$ & $<0.001$ \\
\hline Vertebra & $62(4.1)$ & $23(3.9)$ & 0.806 \\
\hline Pelvis & $6(0.4)$ & $4(0.7)$ & 0.407 \\
\hline Genital region & $5(0.3)$ & $0(0)$ & 0.160 \\
\hline \multicolumn{4}{|l|}{ Location of accident, $n(\%)$} \\
\hline Home & $459(30.5)$ & $192(32.4)$ & 0.389 \\
\hline Street & $793(52.7)$ & $277(46.8)$ & 0.015 \\
\hline School & $2(0.1)$ & $5(0.8)$ & 0.011 \\
\hline Park/playground & $36(2.4)$ & $37(6.2)$ & $<0.001$ \\
\hline Sports field & 29 (1.9) & $5(0.8)$ & 0.077 \\
\hline Workplace & $128(8.5)$ & $50(8.4)$ & 0.965 \\
\hline Unspecified & $58(3.9)$ & $26(4.4)$ & 0.572 \\
\hline
\end{tabular}

*Chi-square test, $p<0.05$ considered significant

in surgical emergency admissions [15]. The inclusion of all kind of trauma patients in our study may have caused this difference in rates. Similar to the studies of Esteban et al.

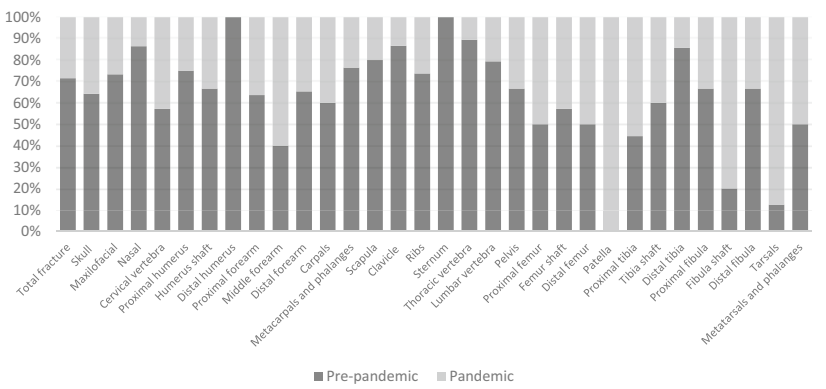

Fig. 2 Fracture locations of the patients during the pre-pandemic and pandemic period

and Göksoy et al., an increase in emergency visits of critical trauma patients was observed in our study $[15,16]$. In this case, it can be said that critically ill trauma patient visits have increased during the pandemic period. Also, a decrease was found in the visits of ESI level 3 and 4 patients in our study. However, the visits of ESI level 5 patients increased. In this case, it can be said that while the emergency visit of trauma patients with moderate urgency decreased, the number of critical and non-urgent (minor) trauma visits increased. During the pandemic period, monitored (where critically ill trauma patients could be followed up) and fasttrack (where minor trauma patients could be evaluated) units should be established for trauma patients.

In the study conducted by Kamine et al., it was found that there was a decrease in the number of hospitalizations and no change in the number of emergency surgery [17]. In the study conducted by Park et al., while hospital admissions increased during the pandemic period, there was no change in

Table 3 Factors affecting the adult trauma visits during COVID-19 outbreak

\begin{tabular}{llll}
\hline & Odds ratio & $\begin{array}{l}95 \% \\
\text { confidence } \\
\text { interval }\end{array}$ & $p$ value \\
\hline Arrival, ambulance & 1.66 & $1.27-2.18$ & $<\mathbf{0 . 0 0 1}$ \\
ESI, level 4 & 0.87 & $0.68-1.11$ & 0.283 \\
Blunt trauma presence & 0.49 & $0.38-0.63$ & $<\mathbf{0 . 0 0 1}$ \\
Dislocation presence & 1.93 & $0.86-1.35$ & 0.063 \\
Fall from high level & 3.00 & $1.68-5.36$ & $<\mathbf{0 . 0 0 1}$ \\
Motorcycle accident & 3.43 & $2.07-5.69$ & $<\mathbf{0 . 0 0 1}$ \\
Multiple trauma & 0.27 & $0.18-0.38$ & $<\mathbf{0 . 0 0 1}$ \\
Upper limb injury & 0.73 & $0.53-0.99$ & $\mathbf{0 . 0 0 5}$ \\
Lower limb injury & 0.99 & $0.71-1.38$ & 0.980 \\
Location of injury, street & 0.80 & $0.64-1.00$ & 0.060 \\
Location of injury, park/play- & 2.37 & $1.43-3.93$ & $\mathbf{0 . 0 0 1}$ \\
$\quad$ ground & & & \\
Disposition, discharge & 0.67 & $0.51-0.89$ & $\mathbf{0 . 0 0 5}$ \\
\hline
\end{tabular}

$p<0.05$ considered significant

$E S I$ emergency severity index 
emergency surgical need [18]. In a study by Cano-Valderrama et al., a reduction in the number of emergency surgery was found [4]. In Göksoy et al.'s study, there was no change in the hospitalizations and surgical needs of the patients [15]. There was no change in the trauma and emergency surgery needs, ICU, and ward admissions of the patients during the pandemic period in our study. In this case, although elective surgeries are postponed during the pandemic period, there is still a need for appropriate ward and ICU beds and operating room conditions for trauma patients. In the management of critical trauma patients and surgical applications, recommendations under pandemic conditions should be followed [19]. In addition, while determining the working areas of trauma surgeons, it should be considered that the visits of critical trauma patients have increased, and there may be an urgent need for surgery. Trauma teams should continue their duties during the pandemic period.

In the studies of Qasim et al. and Andreozzi et al., there was no significant change in the ED LOS during the pandemic period similar to our study [20,21]. In this case, it can be said that the management of trauma patients in our ED is done quickly and effectively as in the pre-pandemic period.

In Andreozzi et al.'s study, patients preferred to arrive at the ED by ambulance at a higher rate similar to our study [20]. Emergency medical services should be supported by additional staff, equipment, and vehicle during the pandemic period.

In the study of Qasim et al., the frequency of penetrating trauma increased, while the frequency of animal bites did not change [21]. In our study, the frequency of penetrating trauma and animal bites increased. There was no change in burns and fracture frequency. Also, there was no significant change in the number of patients consulted to trauma clinics such as general surgery, neurosurgery, cardiovascular surgery, and thoracic surgery. Although all clinical specialties are assigned to combat against COVID-19 during the pandemic period, trauma surgeons are still needed for trauma patients.

While motorcycle accidents decreased by approximately $39 \%$ in Chiba et al.'s study, they increased significantly in our study [22]. During the pandemic period, after the closure of restaurants, our government let only motor courier deliveries for restaurants. Additionally, most of the supermarkets started to motor courier deliveries to reduce social contact and protect against contamination. The increase in the number of motor courier deliveries can be shown as the reason for the increase in motorcycle accidents during the pandemic period. Public information should be provided to the prevention of motorcycle accidents.

In the DiFazio et al.'s study, an approximately $75 \%$ reduction in motor vehicle accidents was found, similar to our study [23]. In addition, pedestrian injuries decreased during the pandemic period. Curfews and intercity travel bans can be shown as the reason for this decrease.

Similar to the study by Andreozzi et al., the multiple trauma frequency was decreased in our study [20]. Similar to the work of Esteban et al., the incidence of upper and lower limb injuries increased during the pandemic period in our study [16]. With the curfew, people started to spend their time at home. This situation can be shown as the reason for the increase in low-energy upper and lower limb injuries and the decrease in multiple traumas.

In the study by Andreozzi et al., the frequency of inhome injuries increased, but there was no change in our study and the study conducted by Zhu et al. [20, 24]. In the study conducted by Bram et al. in pediatric trauma patients, a reduction in in-home injuries was found [11]. It can be said that adult patients are less exposed to inhome trauma in both pandemic and pre-pandemic periods compared to the pediatric population. While the frequency of park injuries increased during the pandemic period, the frequency of injuries on the street decreased. The increase in park injuries may have been caused by the people spending more time in the parks and playgrounds when there is no restriction.

\section{Limitations}

The first limitation of our study is that it was a single-center and retrospective study. Some data were not available due to the retrospective design of the study. The second limitation of our study is that the rabies vaccination program has not yet started in the pre-pandemic period in the study hospital. This program may have caused an imbalance in the number of animal bite cases between groups.

\section{Conclusions}

Despite the decrease in the visit frequency of total adult trauma patients during the pandemic period, the needs for emergency surgery, ICU, and ward admission did not change. The frequency of critical trauma patients increased during the pandemic period. The number of patients consulted to various trauma specialties did not change. Surgeon and bed needs for trauma patients should be taken into consideration while planning for pandemics. Since the use of ambulance increases during the pandemic period, emergency medical services should be supported by additional vehicles, personnel, and equipment. Motorcycle accidents increased during the pandemic period. There is a need for public announcements to prevent motorcycle accidents. 
Author contribution Study concept and design (B.İ., G.B.B., H.D.), acquisition of the data (H.A., M.A.E.), analysis and interpretation of the data (B.İ., G.B.B.), drafting of the manuscript (B.I., G.B.B., H.A., M.A.E., H.D.), critical revision of the manuscript for important intellectual content (B.I.,, G.B.B., H.A., M.A.E., H.D.).

Data availability The data that support the findings of this study are available on request from the corresponding author.

Code availability Not applicable.

\section{Declarations}

Ethics approval The study protocol was approved by the Ethics Committee of Bakırköy Dr. Sadi Konuk Training and Research Hospital (Approval ID: 2021/56; 01/02/2021).

Consent to participate The informed consent could not be obtained because of the retrospective nature of the study.

Consent for publication The consent for publication could not be obtained because of the retrospective nature of the study.

Competing interests The authors declare no competing interests.

\section{References}

1. Cucinotta D, Vanelli M (2020) WHO declares COVID-19 a pandemic. Acta Biomed 91(1):157-160

2. World Health Organization (2021) Coronavirus disease (COVID19) dashboard. https://covid19.who.int/ (Accessed: 30 Augt 2021)

3. İlhan B, Berikol GB, Dogan H (2021) Impact of COVID-19 outbreak on emergency visits and emergency consultations: a crosssectional study. Cureus. 13(3):e14052

4. Cano-Valderrama O, Morales X, Ferrigni CJ et al (2020) Acute care surgery during the COVID-19 pandemic in Spain: changes in volume, causes and complications. A multicentre retrospective cohort study. Int J Surg. 80:157-161

5. Patel R, Hainsworth AJ, Devlin K, Patel JH, Karim A (2020) Frequency and severity of general surgical emergencies during the COVID-19 pandemic: single-centre experience from a large metropolitan teaching hospital. Ann R Coll Surg Eng. 1-6

6. Bres Bullrich M, Fridman S, Mandzia JL et al (2020) COVID-19: stroke admissions, emergency department visits, and prevention clinic referrals. Can J Neurol Sci. 1-4

7. Diegoli H, Magalhaes PSC, Martins SCO et al (2020) Decrease in hospital admissions for transient ischemic attack, mild, and moderate stroke during the COVID-19 era. Stroke 51(8):2315-2321

8. Mafham MM, Spata E, Goldacre R et al (2020) COVID-19 pandemic and admission rates for and management of acute coronary syndromes in England. Lancet 396(10248):381-389

9. Greenhalgh M, Dupley L, Unsworth R, Boden R (2020) Where did all the trauma go? A rapid review of the demands on orthopaedic services at a UK Major Trauma Centre during the COVID-19 pandemic. Int J Clin Pract. e13690
10. Gumina S, Proietti R, Polizzotti G, Carbone S, Candela V (2020) The impact of COVID-19 on shoulder and elbow trauma: an Italian survey. J Shoulder Elbow Surg 29(9):1737-1742

11. Bram JT, Johnson MA, Magee LC et al (2020) Where have all the fractures gone? The epidemiology of pediatric fractures during the COVID-19 pandemic. J Pediatr Orthop 40(8):373-379

12. Nabian MH, Vosoughi F, Najafi F et al (2020) Epidemiological pattern of pediatric trauma in COVID-19 outbreak: Data from a tertiary trauma center in Iran. Injury

13. Ganjali R, Golmakani R, Ebrahimi M, Eslami S, Bolvardi E (2020) Accuracy of the emergency department triage system using the emergency severity index for predicting patient outcome; a single center experience. Bull Emerg Trauma 8(2):115-120

14. Pintado JF, Gibaja W, Vallejos RA et al (2020) How COVID-19 has affected emergent visits to a Latin-American trauma department: experience at a Peruvian national trauma referral center. Injury 51(12):2834-2839

15. Göksoy B, Akça MT, Inanç ÖF (2020) The impacts of the COVID-19 outbreak on emergency department visits of surgical patients. Turkish Journal of Trauma and Emergency Surgery 26(5):685-692

16. Esteban PL, Querolt Coll J, Xicola Martínez M, Camí Biayna J, Delgado-Flores L (2020) Has COVID-19 affected the number and severity of visits to a traumatology emergency department? Bone $\&$ joint open 1(10):617-620

17. Kamine TH, Rembisz A, Barron RJ, Baldwin C, Kromer M (2020) Decrease in trauma admissions with COVID-19 pandemic. West J Emerg Med 21(4):819-822

18. Park C, Sugand K, Nathwani D, Bhattacharya R, Sarraf KM (2020) Impact of the COVID-19 pandemic on orthopedic trauma workload in a London level 1 trauma center: the "golden month." Acta Orthop 91(5):556-561

19. Gok AFK, Eryilmaz M, Ozmen MM et al (2020) Recommendations for trauma and emergency general surgery practice during COVID-19 pandemic. Ulus Travma Acil Cerrahi Derg 26(3):335-342

20. Andreozzi V, Marzilli F, Muselli M et al (2020) The impact of COVID-19 on orthopaedic trauma: a retrospective comparative study from a single university hospital in Italy. Orthop Rev (Pavia) 12(4):8941

21. Qasim Z, Sjoholm LO, Volgraf J et al (2020) Trauma center activity and surge response during the early phase of the COVID-19 pandemic-the Philadelphia story. J Trauma Acute Care Surg 89(4):821-828

22. Chiba H, Lewis M, Benjamin ER et al (2021) "Safer at home": the effect of the COVID-19 lockdown on epidemiology, resource utilization, and outcomes at a large urban trauma center. J Trauma Acute Care Surg 90(4):708-713

23. DiFazio LT, Curran T, Bilaniuk JW et al (2020) The impact of the COVID-19 pandemic on hospital admissions for trauma and acute care surgery. Am Surg 86(8):901-903

24. Zhu W, Li X, Wu Y et al (2020) Community quarantine strategy against coronavirus disease 2019 in Anhui: an evaluation based on trauma center patients. Int J Infect Dis 96:417-421

Publisher's Note Springer Nature remains neutral with regard to jurisdictional claims in published maps and institutional affiliations. 